\title{
Strategies to cope with sodium layer profile variations in laser guide star AO systems
}

\section{Brent L. Ellerbroek}

Brent L. Ellerbroek, "Strategies to cope with sodium layer profile variations in laser guide star AO systems," Proc. SPIE 9148, Adaptive Optics Systems IV, 91482A (21 July 2014); doi: 10.1117/12.2054415

EDent: SPIE Astronomical Telescopes + Instrumentation, 2014, Montréal, Quebec, Canada 


\title{
Strategies for Coping with Sodium Profile Variations in Laser Guide Star AO Systems
}

\author{
Brent L. Ellerbroek \\ TMT Observatory Corporation, 1111 S. Arroyo Pkwy., Suite 200, Pasadena, CA 91105, USA
}

\begin{abstract}
The vertical profile of the mesospheric sodium layer varies significantly on a time scale of one minute. These variations can impact the random and systematic measurement errors of laser guide star Shack-Hartmann wave front sensors, particularly on extremely large telescopes. Sensor performance can be improved by selecting pixel processing weights matched to the sodium layer profile, assuming that the shape of the profile can be measured or estimated in real time.

In this paper we describe the magnitude of these effects for the Thirty Meter Telescope AO system NFIRAOS. We review several existing approaches for measuring or estimating the sodium layer profile in real time. We then describe a new method for estimating the profile directly from the laser guide star wave front pixel intensities themselves, jointly with the subaperture tip/tilt measurements. The algorithm used for this purpose is based upon the multi-frame iterative blind deconvolution algorithm from image post processing: Subaperture tip/tilts and the sodium profile are estimated successively, bootstrapping the estimate of each quantity from the previous estimate of the other. We present promising initial simulation results on the potential performance of the algorithm, and suggest areas for future work.
\end{abstract}

Keywords: Adaptive optics, laser guide stars, wave front sensing

\section{INTRODUCTION}

All of the extremely large telescopes (ELTs) currently under development expect to employ laser guide star (LGS) adaptive optics (AO), and more specifically utilize Shack-Hartmann wave front sensors (WFSs) with artificial guide stars generated in the mesospheric sodium layer. ${ }^{1-3}$ As illustrated in Fig. 1, the subaperture images (or "spots") of a sodium laser guide star will be elongated on account of the nonzero depth of this layer.* This effect is proportional to the telescope aperture diameter, and increases from an observable phenomena on current 8-10 m-class telescopes to a potentially serious wave front error source on ELTs. The structure of the subaperture spots will vary in time as the vertical profile of the sodium layer changes. Wavefront sensing measurement accuracy can be improved, both in terms of sensitivity to measurement noise and systematic biases, if the sodium layer profile is known and properly accounted for in WFS pixel processing algorithms. ${ }^{5}$

Proposed approaches for coping with the impact of sodium layer profile variability have included (i) modulating the output of the guide star laser system to obtain LIDAR-like measurements of the sodium layer profile, ${ }^{6}$ (ii) using a natural guide star (NGS) "truth" WFS (TWFS) to detect biases in the LGS wave front reconstruction, ${ }^{7}$ (iii) dithering the LGS pointing on the sky to estimate the tip/tilt measurement gain of the LGS WFS, ${ }^{7}$ and (iv) suppressing reconstruction of a number of wave front modes which are most sensitive to variations in the sodium layer profile. ${ }^{8}$ In this paper, we propose to estimate the sodium layer profile directly from the LGS WFS pixel intensities themselves, using an algorithm inspired by the "multi-frame iterative blind deconvolution" approach for jointly estimating an unknown object and set of point spread functions from a time series of multiple focal plane images. ${ }^{9}$ The role of the multiple focal plane images is played by the multiple Shack-Hartmann spots; the subaperture tip/tilts (or gradients) correspond to the multiple point spread functions; and the sodium layer

Further author information:

E-mail: brente@caltech.edu, Telephone: 16263951620

*Although the elongation can in principle be eliminated using a guide star laser with the appropriate pulse format and a WFS which "dynamically refocuses" to track the short laser pulse through the sodium layer, ${ }^{4}$ this concept does not appear ready to be implemented in the first generation of ELT LGS AO systems. 


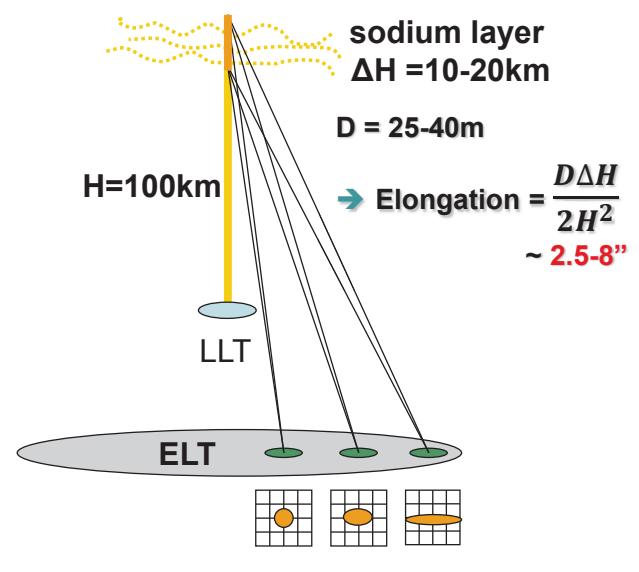

Figure 1. LGS Elongation for Shack-Hartmann WFSs

profile, convolved with the subaperture PSF without elongation effects, is analogous to the unknown object. The subaperture tip/tilts and the sodium layer profile "object" are estimated iteratively until convergence is obtained. In sample test cases using LIDAR measurements of actual sodium layer profiles and simulated LGS WFS pixel intensities for the Thirty Meter Telescope (TMT) AO system NFIRAOS, ${ }^{10}$ the systematic wave front reconstruction error due to uncertainty in the estimate of the sodium layer profile is reduced to $20 \mathrm{~nm}$ RMS or less after 10-20 iterations of the IBD algorithm.

The remainder of this paper is organized as follows. Sec. 2 briefly reviews the impact of sodium layer variability on LGS AO systems, including variations in the integrated column density, mean altitude, and vertical profile. Sec. 3 illustrates the benefits of accounting for the sodium layer profile in LGS wave front sensing, with a performance comparison between the weighted center-of-gravity (WCoG) and matched filter pixel processing algorithms. Sec. 4 outlines some of the prior methods proposed for adapting the matched filter algorithm in real time as the sodium layer profile varies, and then introduces the overall architecture of the new iterative blind deconvolution (IBD) approach. Sec. 5 describes the formal details of the IBD algorithm as currently implemented, and Sec. 6 presents analytical results on its expected error due to WFS measurement noise and (sodium layer profile) fitting error. Sec. 7 presents sample simulation results illustrating the performance and convergence rate of the algorithm. Sec. 8 provides a brief summary, including some ideas for future work. Finally, the Appendix outlines some background information on the WCoG and matched filter pixel processing algorithms studied in this paper.

\section{EFFECTS OF SODIUM LAYER VARIABILITY AND THEIR MITIGATIONS}

The characteristics of the mesospheric sodium layer vary substantially in terms of the integrated density, mean altitude, overall thickness, and vertical profile of the layer. ${ }^{11}$ Variations in the integrated density of the layer directly impact the signal level of the LGS WFS, and can be mitigated (to some extent) by adjusting the gain of the AO control loop to balance the wave front errors due to measurement noise and time delay. This effect is largely independent of telescope aperture diameter $D$.

As is well known, variations in the mean range of the sodium layer introduce focus errors in the LGS WFS measurements, which must sensed and corrected with a focus measurement from a separate NGS WFS. The magnitude of this effect scales as $D^{2}$, meaning that the sampling rate required from this NGS WFS increases with telescope aperture diameter, reaching $10-100 \mathrm{~Hz}$ for an ELT. ${ }^{12}$

Variations in the overall thickness of the sodium layer (e.g., the FWHM of the layer or the interval encompassing $90 \%$ of the layer) directly influence the amount of LGS elongation, and consequently impact the centroid 

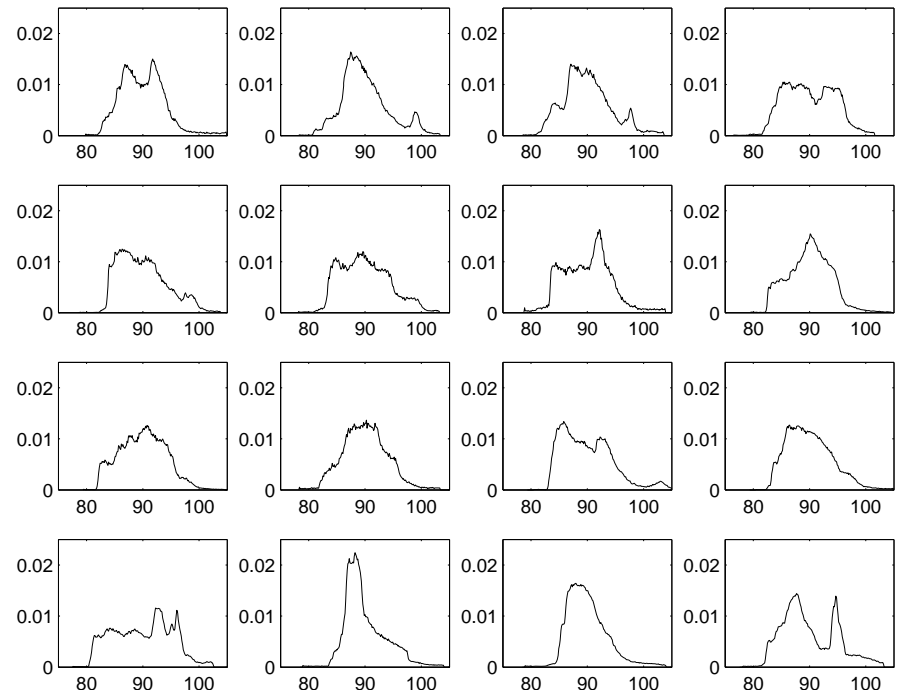

Figure 2. Sixteen sample sodium layer profiles. These are 10-second averages of measurements collected by the LZT Lidar facilty of the University of British Columbia. ${ }^{15}$ They are intended to span the range from typical to somewhat unusual profiles, but are not a statistically representative sample in any quantitative sense. They have been normalized to the same integrated column density (in arbitrary units) and a mean altitude of $90 \mathrm{~km}$.

gain of the LGS WFS and the tip-tilt measurement error due to photon and detector readout noise. These effects are proportional to $D$, and become very significant for ELT aperture diameters. The variations in centroid gain can be addressed by using more than $2 \times 2$ pixels per WFS subaperture, although this has the consequence of increasing pixel read rates and detector read noise. The impact on the measurement error due to noise can be reduced by adapting the pixel processing weights in real time as the thickness of the sodium layer varies, using (for example) a weighted center of gravity (WCoG) or a matched filter processing algorithm. ${ }^{13,14}$

Higher-order variations in the vertical profile of the layer can effect the biases of the LGS WFS measurements (i.e., the instantaneous value of the WFS reference vector), as well as the measurement error due to noise. These variations can be significant, as illustrated by the 16 sample profiles in Fig. 2. There is no simple scaling in $D$ for the effects of these profile variations, since they will depend upon the particular sampling (or under sampling) of the profile variations on the LGS WFS detectors. However, it is fair to say that the effects will be greater for ELTs than for current LGS AO systems, given that magnitude of the LGS elongation for an edge subaperture will increase from about 1 to 4 arc sec.

As stated previously, variations in the measurement biases can in principle be corrected by an independent NGS TWFS or direct, independent measurements of the sodium layer profile. WFS measurement error due to noise can be minimized by adjusting the pixel processing weights to match the structure of the profile, using either independent measurements of the profile or by dithering the LGS pointing on the sky.

The range of different profile shapes illustrated in Fig. 2 suggests that their impact on LGS AO performance may be substantial. We will focus on this effect for the remainder of the paper, assuming that the integrated density and mean altitude of the sodium layer are constant or at least known in advance. In the following section, we first evaluate the potential benefits to WFS performance if the sodium profile is also a priori known, before investigating several options for tracking the profile variations in real time.

\section{MATCHED FILTER VS. WEIGHTED CENTER-OF-GRAVITY PIXEL PROCESSING}

The formal details of the WCoG and matched filter pixel processing algorithms are summarized in Appendix A below. Very briefly, the "matched filter" method is synonymous with noise-weighted least squares, and the pixel processing weights for this algorithm are chosen to minimize the mean-square estimation error due to noise for 
small tip-tilt perturbations around a known reference point. In contrast, the WCoG algorithm uses the standard centroid weights scaled by a windowing function, for example $f(x, y)=\exp \left(-x^{2} / 2 \sigma_{x}^{2}\right) \exp \left(-y^{2} / 2 \sigma_{y}^{2}\right)$. Narrowing the width of the window by reducing the values of $\sigma_{x}$ and $\sigma_{y}$ will reduce the tip-tilt estimation error due to measurement noise, but can also introduce gain variations and estimation biases, particularly for asymmetric or irregular sources such as elongated laser guide stars. Performance is optimized by finding a compromise window width which balances these two competing error sources.

To exercise the matched filter and WCoG pixel processing algorithms on a range of sample data, we have computed LGS WFS subaperture images for the 16 sample sodium profiles in Fig. 2, the LGS WFS parameters listed in Table 1, and a simplified LGS WFS model. This simplified model approximates the projected laser beam on the sky and the point spread function (PSF) for each WFS subaperture as a two-dimensional Gaussian, with a FWHM picked to approximate atmospheric seeing. ${ }^{\dagger}$ We applied the WCoG algorithm to the simulated images with a range of different window widths to compute the tip/tilt estimation biases, reconstructed the biases into a wave front estimation error,$^{\ddagger}$, and computed the RMS value of this error with best-fit focus removed. We removed the focus term to model the correction applied by the tip-tilt-focus NGS WFS, which will sense and correct focus errors an update rate substantially faster than the anticipated variations in the sodium layer profile.

Table 1. AO system parameters for LGS WFS analysis and simulation. The parameters are based upon the TMT first light facility AO system NFIRAOS ${ }^{10}$ and its polar coordinate LGS WFS. ${ }^{16}$

\begin{tabular}{|l|l|}
\hline Aperture diameter, $\mathrm{m}$ & 30 \\
\hline Subaperture width, $\mathrm{m}$ & 0.5 \\
\hline Seeing, arc sec & 0.58 at $\lambda=0.5 \mu \mathrm{m}$ \\
\hline Laser launch location & Center launch \\
\hline WFS pixel width, arc sec & 0.5 \\
\hline Pixels per subaperture & $16 \times 6$ (Polar coordinate geometry) \\
\hline WFS signal level & 900 PDEs per subaperture per frame \\
\hline WFS read noise & 3 electrons per pixel per read \\
\hline
\end{tabular}

Fig. 3 plots the wave front reconstruction biases computed for the WCoG algorithm for a range of different window widths in the radial dimension. The results are plotted as cumulative probability distributions, computed over the 16 different sodium layer profiles illustrated in Fig. 2. According to these results, the FWHM of the centroiding window must be on the order of 20 arc sec or larger to constrain the focus-removed wave front reconstruction bias to about $50 \mathrm{~nm}$ RMS for a majority of the sodium layer profiles.

To compare the noise performance of the matched filter and WCoG algorithms, we used the formulas in Appendix A to compute the increase in the nominal LGS WFS signal level that would be needed for the WCoG approach to achieve the same radial tip/tilt measurement error due to noise as the matched filter algorithm in an edge subaperture. The results are plotted in Fig. 4. For a radial WCoG window width of 20 arc sec or greater, the WCoG method typically requires about 1.6-2.0 times the signal level as the matched filter approach, implying significantly greater laser power to generate brighter guide stars.

The results in Fig.'s 3 and 4 present a dilemma for the WCoG pixel processing algorithm. For many sodium layer profiles, it appears difficult to define a window width which simultaneously achieves satisfactory performance in terms of wave front reconstruction bias and wave front error due to measurement noise. Although some further performance optimization may be possible, the potential benefits of the matched filter are clear. In the remainder of this paper, we will focus on the need to measure or estimate the sodium layer profile in real time to successfully implement the matched filter.

\footnotetext{
${ }^{\dagger}$ The formal details of the model are given in Sec. 5 below.

${ }^{\ddagger}$ On account of the radially symmetrical guide star elongation pattern for TMT NFIRAOS, the wave front reconstruction was performed in one dimension.
} 


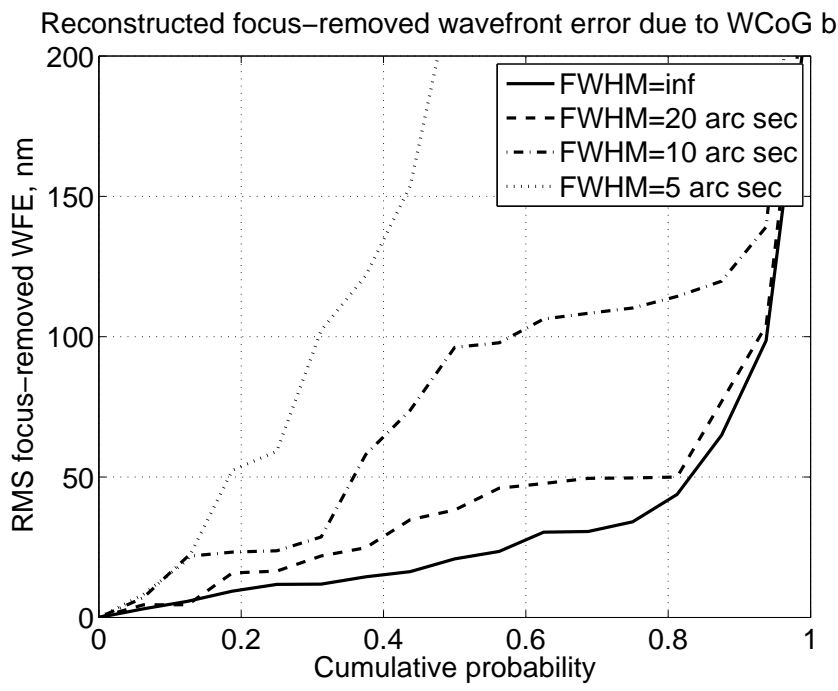

Figure 3. Wavefront reconstruction bias for the WCoG pixel processing algorithm. The probability distributions are computed with respect to the 16 sodium layer profiles in Fig. 2. The listed FWHM values are for the radial direction.

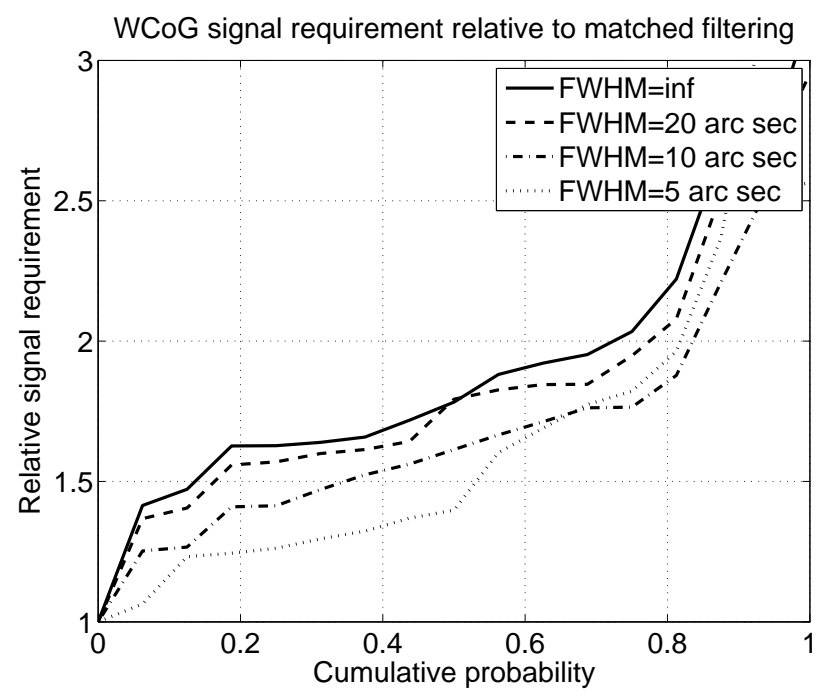

Figure 4. Relative signal level requirements for the WCoG and matched filter pixel processing algorithm to achieve the same radial tip/tilt measurement error due to noise in an edge subaperture. 


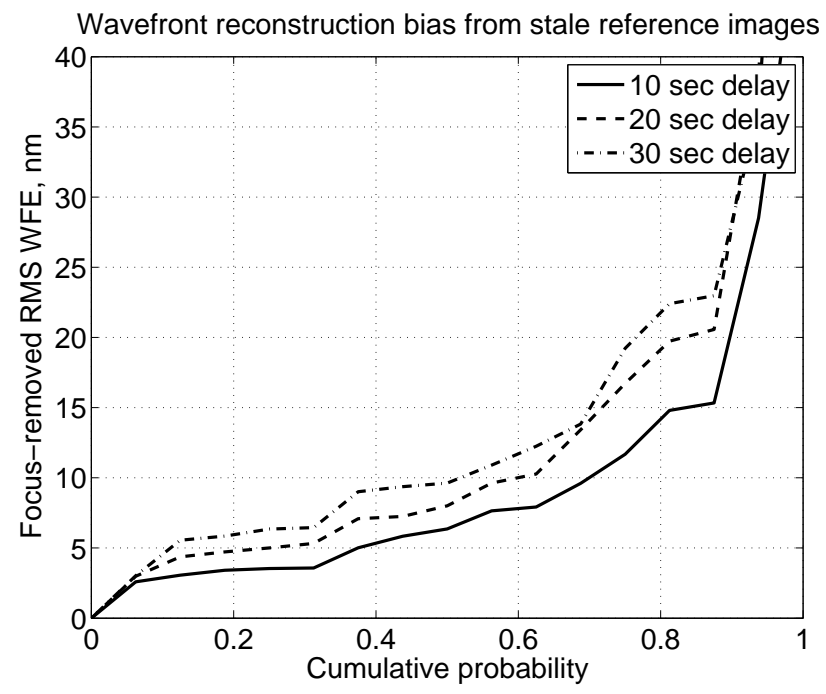

Figure 5. Wavefront reconstruction biases resulting from the use of outdated matched filter coefficients. The probability distributions have been computed for the 16 sodium layer profiles in Fig. 2

\section{OPTIONS FOR UPDATING THE MATCHED FILTER IN REAL TIME}

\subsection{The Required Update Rate}

The 16 sodium layer profiles illustrated in Fig. 2 are actually the first frames of 16 short "movies," each consisting of 30 10-second frames. Fig. 5 plots the focus-removed wave front reconstruction bias if the matched filter computed from a given frame of a movie is applied to LGS WFS measurements computed from a later frame with time delays of 10-30 seconds (i.e., 1-3 frames). A time delay of 10 seconds results in a wave front reconstruction bias of no more than about $15 \mathrm{~nm}$ RMS for 85-90 per cent of the profiles illustrated in Fig. 2. This suggests that a matched filter update rate in the range of $0.3-1.0 \mathrm{~Hz}$ may be sufficient, although a larger sample of sodium profile measurements, possibly with shorter frame times of 2-5 seconds, should be studied to confirm this.

\subsection{Updates Based on LGS Dithering and "Truth" WFS Measurements}

The "Truth" WFS concept refers to a low-bandwidth, relatively high-order NGS WFS which is used to monitor the time-averaged wave front correction from an LGS AO system. This approach can in principle measure a wave front estimation bias due to the sodium layer profile, although the integration time must be long enough to average out the effects of WFS measurement noise, imperfect wave front correction and (for an off-axis NGS) anisoplanatism.

As described in the Appendix, computation of the matched filter pixel processing weights requires knowledge of the quantity $\partial \vec{I} / \partial \vec{\theta}$, the derivatives of the WFS pixel intensities with respect to guide star tip/tilt. These derivatives can be measured by "dithering" the pointing of the laser guide star on the sky a relatively small fraction of a WFS pixel.

Both of these approaches have been demonstrated successfully in simulation and the lab. On account of page constraints, the reader is referred to Ref. 6 for further details.

\subsection{Updates Based on Direct Lidar Measurements}

It may be possible for an LGS AO system to independently measure the sodium layer profile entirely on its own, using the sodium guide star laser itself as a kind of Lidar. If the power of a CW guide star laser is modulated in an irregular but repeated pattern $l(t)$, the guide star return flux $f(t)$ is proportional the convolution of $l(t)$ with the function $g(t)=p(c t / 2) /(c t / 2)^{2}$, where $p$ is the sodium layer profile and $c$ is the speed of light. The function $g$, and consequently the profile $p$, can therefore be determined by first computing the temporal correlation function 


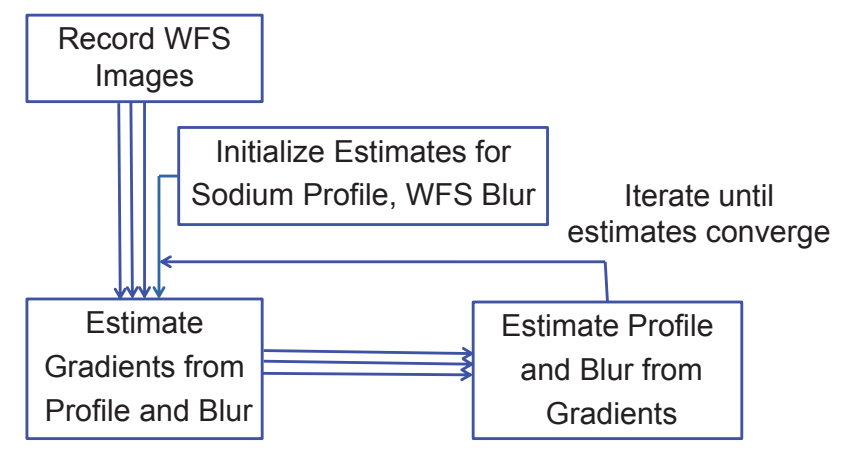

Figure 6. Iterative blind deconvolution concept for jointly estimating the sodium layer profile and sub aperture tip/tilt errors from LGS WFS pixel intensities

$l * f \propto l * l * g$ of the laser power and the guide star return flux, and then filtering out the laser pulse autocorrelation function $l * l$.

This method has been successfully demonstrated in field tests using the ALFA laser at the Calar Alto observatory, ${ }^{6}$ with an average projected laser power of about $1.1 \mathrm{~W}$ modulated $100 \%$ in quasi-random $1 \mu \mathrm{m}$ pulses. Additional tests are now planned using a $20 \mathrm{~W}$-class $\mathrm{CW}$ laser modulated only a few per cent, as this would be the preferred mode of operation in future LGS AO systems.

\subsection{Updates Based on LGS WFS Pixel Intensity Measurements}

Finally, we consider the possibility of estimating the sodium layer profile directly from the LGS WFS pixel intensites themselves. Estimating the sodium layer profile from a single sub aperture image is obviously impossible, given the combined effects of under sampling, LGS WFS measurement noise, sub aperture tip/tilt errors, and uncertainties in the shapes of the sub aperture point spread function and the laser guide star projected on the sky. However, the same sodium layer profile is common to all LGS WFS sub apertures. This may help to average out the effects of measurement noise and the uncertainties in point spread function shapes. Perhaps more importantly, the spatial under sampling in a single WFS sub aperture is potentially reduced by the diversity of the tip/tilt errors in different sub apertures, as well as the differing amounts of guide star elongation depending upon the separation between each sub aperture and the laser launch telescope. We propose that it may be possible to estimate the sodium layer profile and the sub aperture tip/tilt errors jointly to find a solution providing the best fit to the ensemble of measured pixel intensities in all of the WFS sub apertures considered at once.

This concept is based to some extent on the well-known multi-frame iterative blind deconvolution (IBD) algorithm, ${ }^{9}$ which jointly estimates an unknown object $O$ and a number of unknown, aberrated point spread functions $\mathrm{PSF}_{i}$ from the corresponding aberrated, noisy images $\mathrm{PSF}_{i} * O+n_{i}$. The algorithm proceeds by iteratively estimating the point spread functions and then the unknown object, bootstrapping the estimates of each quantity from the previous estimate of the other. In this study, the role of the aberrated point spread functions is taken by the unknown tip/tilts in each WFS sub aperture; the role of the object is taken by the unknown sodium layer profile, convolved by a Gaussian blurring function intended to approximate the convolution of an average sub aperture PSF with the laser beam profile projected on the sky. Fig. 6 is a block diagram illustrating the basic features of the proposed approach.

There are many questions that can be asked about this concept, including:

- What are the formal mathematical details of the algorithm?

- How well can the structure of the sodium layer profile be estimated from the under sampled images?

- What is the sensitivity to WFS measurement noise? 
- What is the sensitivity to LGS WFS modeling errors?

- What are the convergence properties and computational requirements for the algorithm?

- Given that the computational requirements will be large, is there a practical concept for implementing the algorithm in a real-time LGS AO system?

We begin to address some of these issues in the following pages.

\section{FORMULATION OF THE ITERATIVE BLIND DECONVOLUTION METHOD}

\subsection{Subaperture Tip/Tilt Estimation}

The sub aperture tip/tilt estimates are chosen to yield the noise-weighted least-squares fit to the measured pixel intensities at each iteration of the IBD algorithm, based upon a simplified model for the LGS WFS pixel intensities as described below. Briefly, the noise-free signal on pixel number $i$ of WFS sub aperture number $j$ is modeled as

$$
I_{i}^{j}=N_{\mathrm{PDE}}\left(\mathrm{PSF}_{\mathrm{SA}} * \mathrm{PSF}_{\mathrm{LGS}} * \mathrm{DET}_{j} * \mathrm{LGS}_{j}\right)\left(\vec{\theta}_{i}^{j}-\vec{\vartheta}_{j}\right)
$$

where $N_{\mathrm{PDE}}$ is the WFS signal level in photo-detections per sub aperture per frame, $\mathrm{PSF}_{\mathrm{SA}}$ and PSF $\mathrm{LGS}$ are the (simplified) model point spread functions for each LGS WFS sub aperture and the guide star laser projected on the sky, $\mathrm{DET}_{j}$ is the CCD pixel function in sub aperture $j, \mathrm{LGS}_{j}$ is the elongated laser guide star object as viewed from the perspective of sub aperture number $j$, and $\vec{\theta}_{i}^{j}$ is the location of pixel number $i$ in the focal plane of sub aperture number $j .^{\S}$ Finally, the sub aperture tip/tilt is denoted $\vec{\vartheta}_{j}$, and has the effect of translating the LGS image on the WFS pixels.

The sub aperture and LGS point spread functions are modeled as simple Gaussian blurs, namely

$$
\operatorname{PSF}_{\mathrm{SA}}(\vec{\theta})=\operatorname{PSF}_{\mathrm{LGS}}(\vec{\theta})=\exp \left(-\theta^{2} / 2 \sigma^{2}\right),
$$

where $\sigma=\left(\lambda / r_{0}\right) /(2 \sqrt{2 \ln 2})$ has been selected to yield a full-width, half-maximum of $\left(\lambda / r_{0}\right)$ (i.e., atmospheric seeing) for the PSF. The elongated LGS object is given in polar coordinates by

$$
\begin{aligned}
\operatorname{LGS}_{j}\left(\theta_{r}, \theta_{a}\right) & \propto \delta\left(\theta_{a}-\phi_{j}\right) p\left[h_{j}\left(\theta_{r}\right)\right] /\left[h_{j}\left(\theta_{r}\right)\right]^{2}, \\
h_{j}\left(\theta_{r}\right) & =H^{2} \theta_{r} /\left(r_{j}-H \theta_{r}\right),
\end{aligned}
$$

where $\left(r_{j}, \phi_{j}\right)$ is the separation between the launch telescope and sub aperture number $j$ in polar coordinates, the sodium layer profile is denoted $p(h)$, the LGS WFS is focused at altitude $H$, and the function $h_{j}(\theta)$ relates angular elongation in the sub aperture focal plane to altitude in the sodium layer.

Given this simplified forward model, the sub aperture tip/tilts are estimated to minimize the noise-weighted mean-square difference between the measured pixel intensities $m_{i}^{j}$ and the noise-free model values $I_{i}^{j}$, according to the expression

$$
\hat{\vartheta}_{j}=\arg \min _{\vartheta} \sum_{i}\left|I_{i}^{j}-m_{i}^{j}\right|^{2} /\left(I_{i}^{j}+\sigma_{e}^{2}\right) .
$$

Here $\sigma_{e}$ is the RMS detector read noise per pixel per read. At present we are using the standard multi-dimensional, nonlinear MATLAB optimization routine fminsearch to minimize Eq. (5), and have not yet investigated how to improve computational efficiency.

\footnotetext{
$\S$ The locations and the orientation of the pixels are different in each sub aperture for the polar coordinate CCD geometry.
} 


\subsection{Sodium Layer Profile and Seeing Estimation}

Eq. (1) above indicates the the model for the (noise-free or noisy) LGS WFS pixel intensities is linear in the sodium layer profile $p$. Therefore we may write

$$
m=G[\operatorname{PSF}(\sigma), \vartheta] p+n,
$$

where the linear transformation $G$ depends upon the seeing parameter $\sigma$ and the values of the sub aperture tip/tilts. Once the values of the tip/tilts have been estimated as describe above, the estimation of $\sigma$ and $p$ is itself an iterative process. Inverting this equation robustly has not been straightforward (at least for us), since (i) the matrix $G$ is highly ill-conditioned, and (ii) it is also necessary to estimate the seeing parameter $\sigma$ in addition to $p$. The approach described below has proven reasonably successful, although more work is needed to improve its computational efficiency.

For each candidate value of $\sigma$, an estimate $\hat{p}(\sigma)$ is selected to obtain the best noise-weighted fit to the measured pixel intensities using a constrained, regularized estimator. Determining the value $\hat{\sigma}$ which optimizes this fit then reduces to a one-dimensional, nonlinear optimization problem. More formally,

$$
\begin{aligned}
\hat{p}(\sigma) & =\arg \min _{p \in \Omega}\left\{\|m-G[\operatorname{PSF}(\sigma), \hat{\vartheta}] p\|_{I}^{2}+\alpha p^{T} W p\right\}, \\
\hat{\sigma} & =\arg \min _{\sigma}\|m-G[\operatorname{PSF}(\sigma), \hat{\vartheta}] \hat{p}(\sigma)\|_{I}^{2} .
\end{aligned}
$$

Here the set $\Omega$ defines the constraints imposed on the admissible values of $p$, and the quantities $\alpha$ and $W$ describe the regularization which is applied. The vector norm $\|\bullet\|_{I}$ is weighted according the noise in the prior estimate of the LGS WFS pixel intensities, namely

$$
\begin{aligned}
\|f\|_{I}^{2} & =\sum_{i, j}\left(f_{i}^{j}\right)^{2} /\left(I_{i}^{j}+\sigma_{e}^{2}\right) \\
& =f^{T} \Sigma_{n}^{-1} f,
\end{aligned}
$$

where the diagonal matrix $\Sigma_{n}$ is the covariance matrix of the modeled measurement noise $n$.

Two different variants of this approach have been investigated, described by different choices of the quantities $\Omega, \alpha$, and $W$. The first variant is the truncated singular value decomposition (SVD) noise-weighted pseudoinverse, which in this notation is defined by

$$
\Omega_{1}=\operatorname{span}\left\{\vec{v}_{1}, \ldots, \vec{v}_{M}: V \Lambda V^{T}=G^{T} \Sigma_{n}^{-1} G\right\} .
$$

Here $V$ and $\Lambda$ are unitary and diagonal matrices, respectively, and the vectors $\vec{v}_{k}$ are the first $M$ columns of $V$ corresponding to the largest $M$ elements of $\Lambda$. No explicit regularization term has been used with this definition of $\Omega$ (i.e., $\alpha=0$ ) since the truncation of the pseudo-inverse is itself a form of regularization.

The second variant is based upon the constraints

$$
\Omega_{2}=\left\{p: 0 \leq p \leq 1, \sum_{k} p_{k}=1\right\} .
$$

These constraints insure that the profile estimate $\hat{p}$ makes sense physically, but some sort of regularization is still needed because $G$ remains ill-conditioned on this set. To insure that $\hat{p}$ is smoothly varying, we have selected $W$ so that $p^{T} W p=(\Delta p)^{T}(\Delta p)$, where $\Delta p$ is a vector of the point-to-point finite differences of the profile $p$ (i.e., $\left.\Delta p_{i}=p_{i+1}-p_{i}\right)$. This approach obtains a good fit to the true value of the sodium layer profile in the sample problems simulated in Sec. 7 below.

\footnotetext{
"Also, the factor $\alpha$ has been selected so that the magnitude of the regularization term in Eq. (7) is on the order of 0.001-0.01 times the size of the fit-to-data term.
} 


\section{ESTIMATES FOR NOISE AND FITTING ERRORS}

An initial, best-case indication of how well the sodium layer profile might be estimated using the IBD algorithm can be obtained by assuming that (i) the WFS sub aperture tip/tilts $\vartheta_{j}$ are known a priori, (ii) the seeing parameter $\sigma$ is also known, and (iii) the simplified model for the LGS WFS pixel intensities (Eq. (6)) is actually correct. In this case, one application of Eq. (7) yields the constrained, noise-weighted least squares estimate of the sodium layer profile. The estimate is not exact due to WFS measurement noise and the constraints imposed on the permissible solution space for $\hat{p}$.

The performance of the truncated SVD algorithm under these conditions is easier to evaluate. The estimate of the sodium layer profile can be written in the form

$$
\hat{p}=\sum_{i}^{M}\left(c_{i}+e_{i}\right) \vec{v}_{i},
$$

where the variables $c_{i}$ represent the noise-free estimates of the modal coefficients and the quantities $e_{i}$ are the perturbations to these estimates due to WFS measurement noise. These two quantities are given by the expressions

$$
\begin{aligned}
c_{i} & =\Lambda_{i i}^{-1} \vec{v}_{i}^{T} G^{T} \Sigma_{n}^{-1} I \\
& =\vec{v}_{i}^{T} p \\
e_{i} & =\Lambda_{i i}^{-1} \vec{v}_{i}^{T} G^{T} \sigma_{n}^{-1} n
\end{aligned}
$$

where the second line of Eq. (13) follows because the SVD modes $v_{i}$ are orthonormal and $V^{T} \Lambda V=G^{T} \Sigma_{n}^{-1} G$. Since the measurement noise is uncorrelated with $p$, the expected value of the mean-square estimation error can be written as

$$
\left\langle|p-\hat{p}|^{2}\right\rangle=\left(p^{T} p-\sum_{i=1}^{M} c_{i}^{2}\right)+\sum_{i=1}^{M} \Lambda_{i i}^{-1} .
$$

The term within parenthesis is the fitting error, which decreased as $M$ increases. The second summation describes the estimation error due to WFS measurement noise, which increases with increasing $M$.

Fig. 7 plots the RMS sodium layer profile estimation error, normalized by division by $\sqrt{p^{T} p}$, as a function of the number of SVD modes used in the estimate. Results are plotted as cumulative probabilities with respect to the 16 sodium layer profiles illustrated in Fig. 2. The trade off between the estimation errors due to noise and fitting error is readily apparent, with the best results obtained using 20-30 SVD modes. Using the optimal number of modes, the sodium layer profile can be estimated with a residual error of about 10 per cent due to the combined effect of these error sources.

However, it must be recalled that this is a best-case estimate for these error terms under ideal conditions. A somewhat more comprehensive performance assessment of the IBD algorithm is described in the following section.

\section{INITIAL SIMULATION RESULTS}

We have performed two small sets of simulations for several of the measured sodium layer profiles illustrated in Fig. 2. For the first set of simulations, the LGS WFS pixel intensities have been computed using the simplified PSF model given by Eq. (2) above. The second set used LGS WFS pixel intensities computed by the AO simulation code MAOS ${ }^{17}$ for the TMT AO system NFIRAOS. ${ }^{10}$ The truncated SVD method worked extremely well in the first set of simulations, but produced highly nonphysical estimates of the sodium layer profile in the second set. We attribute this to the model mismatch between Eq. (2) and the higher fidelity PSFs computed in MAOS, which include physical optics effects and time-varying wave front aberrations. In comparison, the constrained, regularized estimator described by Eq. (11) continues to give good results with the simulated WFS data from MAOS. We are hopeful that these results can be further improved by adjusting the regularization term in Eq. (7), and defining an appropriate stopping criteria for the IBD iterations. 


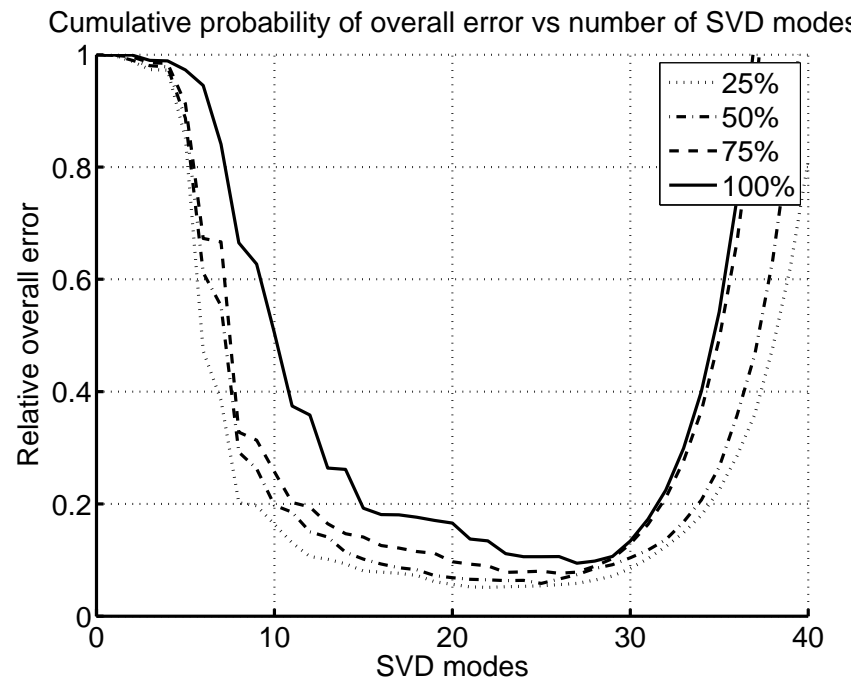

Figure 7. Relative RMS sodium profile estimation error for the truncated SVD variant of the IBD algorithm, as a function of the number of modes utilized. The indicated per centages are cumulative probabilities evaluated over the 16 sodium layer profiles illustrated in Fig. 2.
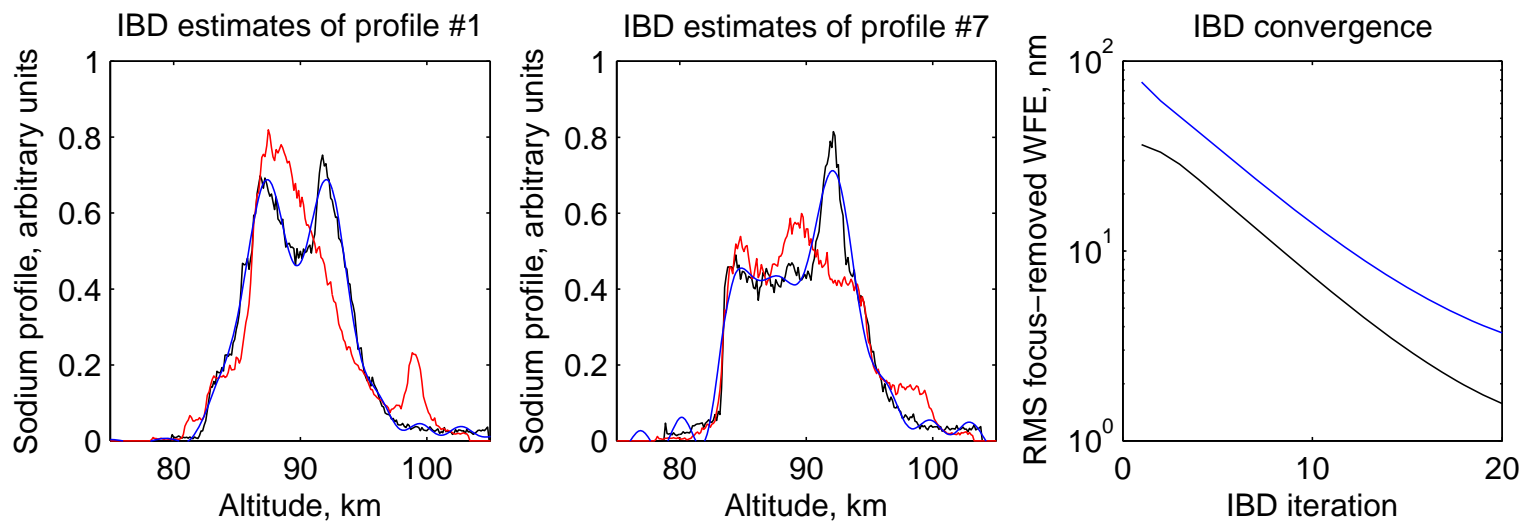

Figure 8. Sample simulation results for the truncated SVD method applied to LGS WFS pixel intensities computed using the simplified PSF model described by Eq. (6) above. The left-hand and center panels of the figure illustrate two sample sodium layer profiles (black), the initial profile estimate (red), and the profile estimate after 10 IBD iterations (blue). The right-hand profile plots the RMS focus-removed wave front estimation bias due to the error in the sodium profile estimate vs. the number of IBD iterations for profile 1 (black) and profile 7 (blue).

Fig. 8 illustrates the results from the first set of simulations on two sample sodium layer profiles. The estimates of the two profiles are qualitatively very accurate after 10 IBD iterations, although they show some modest negative oscillations near their boundaries. These negative values are nonphysical, but they occur because there are no non-negativity constraints in the definition of the SVD modes. The right-hand panel of the figure plots the convergence history of the IBD iterations. After 10 [20] iterations, the RMS focus removed wave front reconstruction bias is in the range of $10-20 \mathrm{~nm}[2-4 \mathrm{~nm}]$ for the two sample profiles simulated. Evidently, the oscillations in the profile estimate do not introduce appreciable wave front reconstruction errors.

This situation changes in the higher-fidelity simulations, using LGS WFS pixel intensities computed with the AO simulation MAOS.\| The truncated SVD method now generates sodium layer profile estimates with much

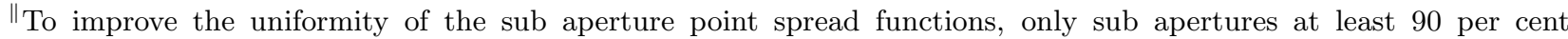
illuminated were used to estimate the sodium layer profile. This is a bit more than 2300 of the 2700 sub apertures in the
} 

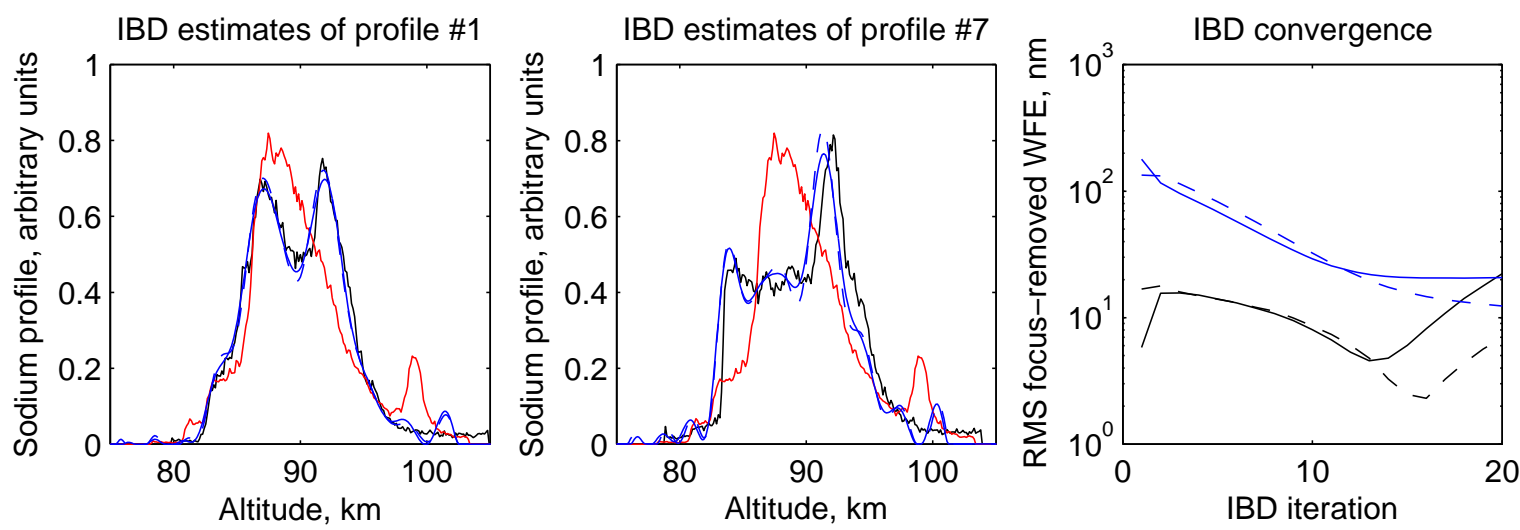

Figure 9. Sample simulation results for the constrained, regularized estimator, applied to LGS WFS pixel intensities computed using the AO simulation code MAOS. The left-hand and center panels of the figure illustrate two sample sodium layer profiles (black), the initial profile estimate (red), and the profile estimate after 20 IBD iterations from a single frame of LGS WFS pixel intensities (blue, dashed), and the estimate after 20 iteration using intensities averaged over 80 frames of WFS measurements (blue, solid). The right-hand profile plots the RMS focus-removed wave front estimation bias due to the error in the sodium profile estimate vs. the number of IBD iterations for profile 1 (black) and profile 7 (blue).

larger oscillations, about 10-20 times greater than the peak-to-valley range of the sodium layer profile itself! As illustrated in Fig. 9, however, the performance of the constrained, regularized estimator remains generally satisfactory for this case, achieving RMS focus-removed wave front estimation biases of about $20 \mathrm{~nm}$ or smaller. However, several aspects of the convergence time histories plotted in the right-hand panel of the figure remain unexplained and/or surprising: (i) the initial rate of convergence is fairly slow, (ii) the performance achieved using instantaneous LGS WFS pixel intensities is as least as good as for time-averaged intensities, and (iii) the estimate eventually begins to diverge for profile number 1. Evidently, more work is needed to accelerate the rate of convergence, define a stopping criteria for the IBD iterations, and perhaps improve the forward model for the LGS WFS.

\section{SUMMARY}

The vertical profile of the mesospheric sodium layer changes significantly on the time scale of one minute. Optimizing the LGS WFS pixel processing weights for the current value of the profile can significantly reduce the power required for guide star laser systems on ELTs, and failing to properly account for the profile can introduce significant wave front reconstruction biases. Several different promising approaches to measuring or estimating the sodium layer profile in real time for LGS AO systems have been previously suggested. In this work, we suggest and investigate the feasibility of estimating the sodium layer profile directly from the measured LGS WFS pixel intensities themselves.

We propose to estimate the sodium layer profile (as well as the atmospheric seeing) jointly with the values of the wave front tip/tilt on each WFS sub aperture. The quantities are estimated sequentially in an iterative algorithm based upon the multi-frame iterative blind deconvolution (IBD) method from image post-processing. An idealized performance analysis based upon a truncated SVD approach indicates that the profile estimation errors due to WFS measurement noise and fitting error (from using a finite number of SVD modes) are acceptably small.

Work is still ongoing to characterize and optimize sodium layer profile estimation accuracy when the IBD algorithm is tested on simulated LGS WFS pixel intensities including diffraction and atmospheric turbulence effects. Preliminary results on several sample sodium layer profiles indicate that relatively accurate estimates can be obtained, corresponding to focus-removed wave front estimation biases of $20 \mathrm{~nm}$ or less due to the profile

NFIRAOS LGS WFS pupil. 
estimation errors. More work is needed to (i) evaluate performance over a wider range of sodium profiles, (ii) accelerate the convergence rate and reduce the computation requirements of the iterative algorithm, (iii) define a stopping criteria for the iterations, and (iv) investigate how the algorithm might be implemented as a background task in the real-time controller for an ELT LGS AO system.

\section{ACKNOWLEDGMENTS}

Angel Otarola provided the sample sodium layer measurements from the LZT Lidar facility. Lianqi Wang provided the simulated NFIRAOS LGS WFS pixel intensities used in Sec. 7.

The TMT Project gratefully acknowledges the support of the TMT partner institutions. They are the Association of Canadian Universities for Research in Astronomy (ACURA), the California Institute of Technology, the University of California, the National Astronomical Observatory of Japan, the National Astronomical Observatories of China and their consortium partners, and the Department of Science and Technology of India and their supported institutes. This work was supported as well by the Gordon and Betty Moore Foundation, the Canada Foundation for Innovation, the Ontario Ministry of Research and Innovation, the National Research Council of Canada, the Natural Sciences and Engineering Research Council of Canada, the British Columbia Knowledge Development Fund, the Association of Universities for Research in Astronomy (AURA), and the U.S. National Science Foundation.

\section{APPENDIX A. WEIGHTED CENTER-OF-GRAVITY AND MATCHED FILTER PIXEL PROCESSING FOR ELONGATED LGS}

The WCoG method for computing the sub aperture tip/tilt estimate $\vartheta$ from the measured pixel intensities $m_{i}$ is described by the expression

$$
\vartheta_{k}=\sum_{i}\left(w_{i} m_{i} \theta_{i, k}\right) / \sum_{i} w_{i} m_{i}
$$

where $k=1$ or 2 is the component of the tip/tilt estimate, $\vec{\theta}_{i}$ denotes the coordinates of pixel number $i$ in the focal plane, and the windowing function $w_{i}$ is given by

$$
w_{i}=\exp \left(-\theta_{i, 1}^{2} / 2 \sigma_{1}^{2}\right) \exp \left(-\theta_{i, 2}^{2} / 2 \sigma_{2}^{2}\right) .
$$

The use of two separate window widths for the tip and tilt components is useful for elongated laser guide stars, particularly for a WFS based upon the polar coordinate CCD array. ${ }^{16}$ The mean-square error in the WCoG tip/tilt estimate due to measurement noise takes the form

$$
\left\langle\left(\vartheta_{k}-\left\langle\vartheta_{k}\right\rangle\right)^{2}\right\rangle \approx \frac{\sum_{i} w_{i}^{2} \theta_{i, k}^{2}\left(I_{i}+\sigma_{e}^{2}\right)}{\left(\sum_{i} w_{i} I_{i}\right)^{2}},
$$

where $I_{i}$ is the noise-free intensity on pixel number $i$ in units of photodetections per frame, and $\sigma_{e}$ is the detector read noise.

The formula for matched filter pixel processing is more easily described in matrix notation:

$$
\vec{\vartheta}=\left[(\partial \vec{I} / \partial \vec{\theta})^{T} \Sigma_{n}^{-1}(\partial \vec{I} / \partial \vec{\theta})\right]^{-1}(\partial \vec{I} / \partial \vec{\theta})^{T} \Sigma_{n}^{-1}(\vec{m}-\vec{I}),
$$

where $\Sigma_{n}^{-1}$ is the (diagonal) covariance matrix of the WFS measurement noise. Assuming that the noise-free pixel intensities I are bilaterally symmetric, as they are for the polar coordinate CCD, the formulas for the tip and tilt estimates decouple and simplify to the form

$$
\vartheta_{k}=\left[\sum_{i} \frac{\left(\partial I_{i} / \partial \theta_{k}\right)^{2}}{\left(I_{i}+\sigma_{e}^{2}\right)}\right]^{-1} \sum_{i} \frac{\left(\partial I_{i} / \partial \theta_{k}\right)}{\left(I_{i}+\sigma_{e}^{2}\right)}\left(m_{i}-I_{i}\right) .
$$

Finally, the mean-square tip/tilt estimation errors due to measurement noise are given by the formula

$$
\left\langle\left(\vartheta_{k}-\left\langle\vartheta_{k}\right\rangle\right)^{2}\right\rangle=\left[\sum_{i} \frac{\left(\partial I_{i} / \partial \theta_{k}\right)^{2}}{\left(I_{i}+\sigma_{e}^{2}\right)}\right]^{-1} .
$$




\section{REFERENCES}

[1] Diolaiti, E., "Overview of the E-ELT MCAO module project," in [Proceedings of the Third AO4ELT Conference], Esposito, S. and Fini, L., eds., Firenze (2013).

[2] Conan, R. et al., "The Giant Magellan Telescope Laser Tomography Adaptive Optics System," in [Proceedings of the Third AO4ELT Conference]. Esposito, S. and Fini, L., eds., Firenze, (2013).

[3] Ellerbroek, B.L., "Adaptive Optics for the Thirty Meter Telescope," in [Proceedings of the Third AO4ELT Conference]. Esposito, S. and Fini, L., eds., Firenze (2013).

[4] Rabien, S. and Ziegleder, J., "Dynamical refocusing laser guide stars with membrane mirrors," in [Adaptive Optics Systems III], Ellerbroek, B., Marchetti, E. and Veran, J.-P., eds., Proc SPIE 8447, 84474P (2012).

[5] Gratadour, D., Gendron, E., and Rousset, G., "Intrinsic limitations of shack-hartmann wavefront sensing on an extended laser guide source," J. Opt. Soc. Am. A 27, 171-181 (2010).

[6] Butler, D. J. et al., "Measuring the Absolute Height and Profile of the Mesospheric Sodium Layer using a Continuous Wave Laser," Astronomy and Astrophysics 403, 775-785 (2003).

[7] Lardiere, O., Conan, R., Bradley, C., Jackson, K., and Herriot, G., "A laser guide star wave front sensor bench demonstrator for TMT," Optics Express 16, 5527-5543 (2008).

[8] Van Dam, M., "Aberrations induced by side-projected laser guide stars in laser tomography adaptive optics systems," in [Proceedings of the Second AO4ELT Conference]. Veran, J.-P., Fusco, T., and Clenet, Y., eds., Victoria (2011).

[9] Sheppard, D.G., Hunt, B.R, and Marcellin, M.W., "Iterative multi-frame super resolution algorithms for atmospheric-turbulence-degraded imagery," J. Opt. Soc. Am. A 15, 978-992 (1998).

[10] Herriot, G. et al., "TMT NFIRAOS: adaptive optics system for the Thirty Meter Telescope," in [Adaptive Optics Systems III], Ellerbroek, B., Marchetti, E. and Veran, J.-P., eds., Proc SPIE 8447, 84471M (2012).

[11] Pfrommer, T. and Hickson, P., "Properties and dynamics of mesospheric sodium and the impact on sodium LGS AO systems," in [Proceedings of the Second AO4ELT Conference]. Veran, J.-P., Fusco, T., and Clenet, Y., eds., Victoria (2011).

[12] Herriot, G. al., "Focus errors from tracking sodium layer altitude variations with laser guide star adaptive optics for the Thirty Meter Telescope," [Advances in Adaptive Optics II], Ellerbroek, B. and Bonaccini Calia, D., eds., Proc SPIE 6272, 84471I (2006).

[13] Schreiber, L. et al., "Laser guide stars for extremely large telescopes: efficient Shack-Hartmann wave front sensor design using the weighted centre-of-gravity algorithm," MNRAS 396, 1513-1521 (2009).

[14] Gilles, L. and Ellerbroek, B., "Shack Hartmann wave front sensing with elongated sodium laser beacons: centroiding versus matched filtering," Appl. Opt., 45, 6568-6576 (2006)

[15] Hickson, P. et al., "The Large Zenith Telescope: A 6 m Liquid-Mirror Telescope," PASP, 119, (2007).

[16] Adkins, S., "Measured performance of the prototype polar coordinate CCD array," in [Adaptive Optics Systems III], Ellerbroek, B., Marchetti, E. and Veran, J.-P., eds., Proc SPIE 8447, 84470R (2012).

[17] Wang, L. and Ellerbroek, B., "Fast End-to-End Multi-Conjugate AO Simulations Using Graphical Processing Units and the MAOS Simulation Code," in [Proceedings of the Second AO4ELT Conference]. Veran, J.-P., Fusco, T., and Clenet, Y., eds., Victoria (2011). 